\title{
Sistema Didáctico de Control de Presión
}

Saida M. Charre-lbarra, Janeth A. Alcalá-Rodríguez, Norberto López-Luiz, Miguel A. Durán-Fonseca Universidad de Colima, km 20 Carretera Manzanillo-Barra, Manzanillo, Colima-México. (e-mail:saidacharre@hotmail.com)

Recibido May. 20, 2014; Aceptado Jun. 27, 2014; Versión final recibida Ago. 27, 2014

\begin{abstract}
Resumen
Se presenta y discute un sistema de control de presión didáctico en forma de arquitectura abierta. Se instaló un controlador digital configurable y además se elaboró un controlador virtual, integrando software y hardware para crear un sistema flexible y abierto a la evolución. La estación de trabajo didáctica está diseñada para desarrollar prácticas y proyectos de investigación con diferentes controladores y sobre todo sus componentes se pueden sustituir por otros, únicamente con la condición de que manejen las señales estándares de instrumentación, con el fin de que no llegue a ser obsoleta. Equipo de laboratorio de este tipo permite comprender y conceptualizar en mejor forma los eventos que ocurren en procesos industriales y explorar los efectos de las modificaciones, estimulando la creatividad en los estudiantes.
\end{abstract}

Palabras clave: arquitectura abierta, estación de trabajo didáctica, controlador virtual, presión, PID

\section{A Didactic Pressure Control System}

\begin{abstract}
This paper discusses a didactic pressure control system operating with open architecture. A digital controller was used and a virtual controller was elaborated. Both software and hardware were integrated to create a flexible system which can be easily updated. The teaching workstation is designed to develop practices and research projects with different controllers, where components can be replaced with others, considering the signals and instrumentation standards that are used, to avoid that this becomes obsolete. The teaching workstation allows to understand and to better conceptualize the events that happen with different industrial processes, as well as to explore the effects of the modifications, stimulating creativity in students.
\end{abstract}

Keywords: open architecture, teaching workstation, virtual controller, pressure, PID 


\section{INTRODUCCIÓN}

En la actualidad todas las industrias desean ser competitivas en el mercado y buscan reducir sus costos de fabricación, mientras que simultáneamente mejoran su calidad, la fiabilidad, la modernidad y la durabilidad de sus productos. Para lograr estos objetivos, se requieren métodos apropiados de administración de producción y optimización, lo cual es imposible sin la aplicación del control automático y la robótica en las tecnologías del proceso. La implementación de las tecnologías se basa en el conocimiento del control automático de procesos continuos, así como de algunos de sus parámetros fundamentales, tales como: cambios de temperatura, contenidos de materiales líquidos en tanques, presión en sistemas, intensidad de luz, velocidad de rotación, etc. Por lo tanto es necesario enseñar a los estudiantes las bases de control y automatización, reglas del funcionamiento de sistemas de sensores, reguladores y sistemas operativos (Świder et al., 2006).

La educación en sus diferentes áreas de la ingeniería demanda la existencia de laboratorios con el fin de proporcionar a los estudiantes conocimiento de calidad y habilidades prácticas en el campo de control de procesos de la vida real (Matijević et al., 2014). El avance de las tecnologías computacionales e informáticas ha dado paso a un nuevo horizonte en el aprendizaje y la enseñanza de prácticas de laboratorio en todo el mundo. A demás de los tradicionales ejercicios prácticos de laboratorio, los del tipo virtual-simulado están jugando un papel cada vez más dominante (Alam et al., 2014). La simulación en ambientes virtuales puede ser una poderosa herramienta para situar a los estudiantes en escenarios "prácticos" a los que difícilmente podrían acceder en la realidad, permitiendo establecer un mayor grado de equidad en el proceso de enseñanza (Fredes et al., 2012).

La combinación de un entorno interactivo dotado de textos, imágenes y simulaciones promueve la participación activa de los estudiantes en su propio proceso de aprendizaje. La utilización de las nuevas tecnologías ofrece una nueva posibilidad en la formación de los profesionales en las diferentes especialidades de la ingeniería (Márquez y Cárdenas, 2008). Por otra parte, actualmente la mayoría de las universidades en los países en desarrollo están bajo una presión financiera debido a los recortes de fondos de los gobiernos en sectores de la educación. Lo que afecta significativamente a los programas de ingeniería, obligándolos a encontrar formas alternativas para la cumplir con la educación práctica debido a la reducción o reemplazo de laboratorios físicos (Alam et al. 2014).

Atendiendo a la necesidad de una formación de calidad de ingenieros en el área de control y la situación actual económica, en el presente trabajo se diseña e implementa un sistema de control de presión didáctico en forma de arquitectura abierta. Por supuesto que existen varios equipos didácticos disponibles en el mercado (Festo-didactic; Tecquipment), pero el diseño de su arquitectura permite que pueda ser modificado conforme avanza la tecnología o según las necesidades que presenten los programas educativos, de manera que se realicen por ejemplo prácticas para el diseño de diferentes algoritmos de control. Ventaja que presentaría debido a que en el mercado la mayoría de los equipos didácticos existentes, tienen el inconveniente de ser sistemas cerrados, difíciles de modificar y por lo tanto pueden llegar a ser obsoletos, y además presentan un alto costo (Guevara et al., 2009). Al final del artículo se presentan las respuestas transitorias obtenidas que demuestran el funcionamiento del sistema.

\section{DESARROLLO E IMPLEMENTACIÓN DEL SISTEMA DE CONTROL DIDÁCTICO}

Dentro de los procesos industriales la medición y el control de la variable presión se hacen indispensables para lograr obtener una producción continua y contar con condiciones de operación seguras. Cualquier recipiente o tubería tiene una presión máxima de operación y sobrepasarla puede ocasionar la destrucción no solo del mismo equipo sino también la del adyacente y poner al personal en una situación de riesgo, principalmente cuando están implícitos fluidos inflamables o corrosivos. Para tales aplicaciones, el control y la medición con gran precisión para la variable presión son tan importantes como la seguridad extrema.

A partir del diagrama a bloques de un control en lazo cerrado mostrado en la Figura 1, se plantea el esquema del sistema de control de presión didáctico (Figura 2). La Figura 2 corresponde al sistema de control de presión didáctico en arquitectura abierta. Este sistema consta de dos tanques, el tanque 1 tiene una capacidad de $13 \mathrm{Kg} / \mathrm{cm}^{2}$ y la capacidad del tanque 2 es de $6 \mathrm{Kg} / \mathrm{cm}^{2}$. El sistema tiene una válvula para simular el consumo que puede existir en un proceso continuo. Una vez energizado el sistema se necesita tener conectado un compresor a la válvula 1, ya que a través de ésta se alimenta el flujo de aire hacia el sistema, se debe asegurar que la válvula 3 de desfogue este cerrada, teniendo esto en cuenta se procede a abrir las válvulas 2, 4, 5 y 6, así como las válvulas que alimentan cualquiera de los dos tanques, el transmisor le enviará una señal de corriente proporcional a la presión del sistema al controlador, el cual hace una comparación entre el punto de ajuste y el valor actual de la variable del proceso, para enviar una señal correctora a la válvula de control. 


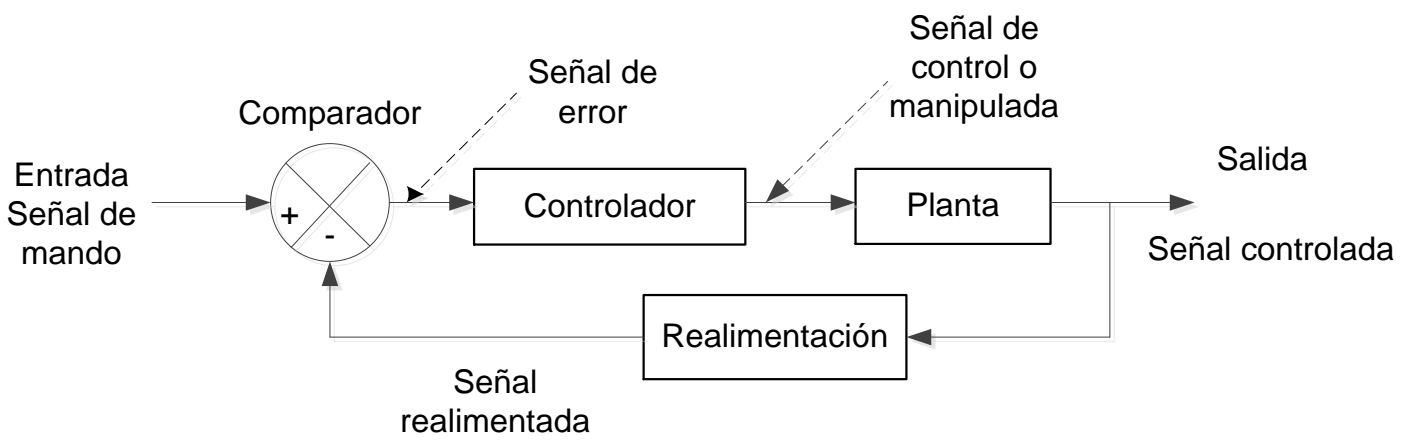

Fig. 1: Diagrama a bloques de un sistema de control en lazo cerrado.

Como sistema en arquitectura abierta, se tiene la opción de poder controlar la variable presión haciendo uso de un controlador digital configurable, un controlador virtual u otro tipo de controladores elegidos 0 diseñados por el usuario, que manejen la señal estándar de instrumentación (4-20 mA) (Pallas, 2008). Para lo cual se instala un tablero de conexiones que proporciona las señales de salida del transmisor y controlador; y las señales de entrada al controlador y válvula.

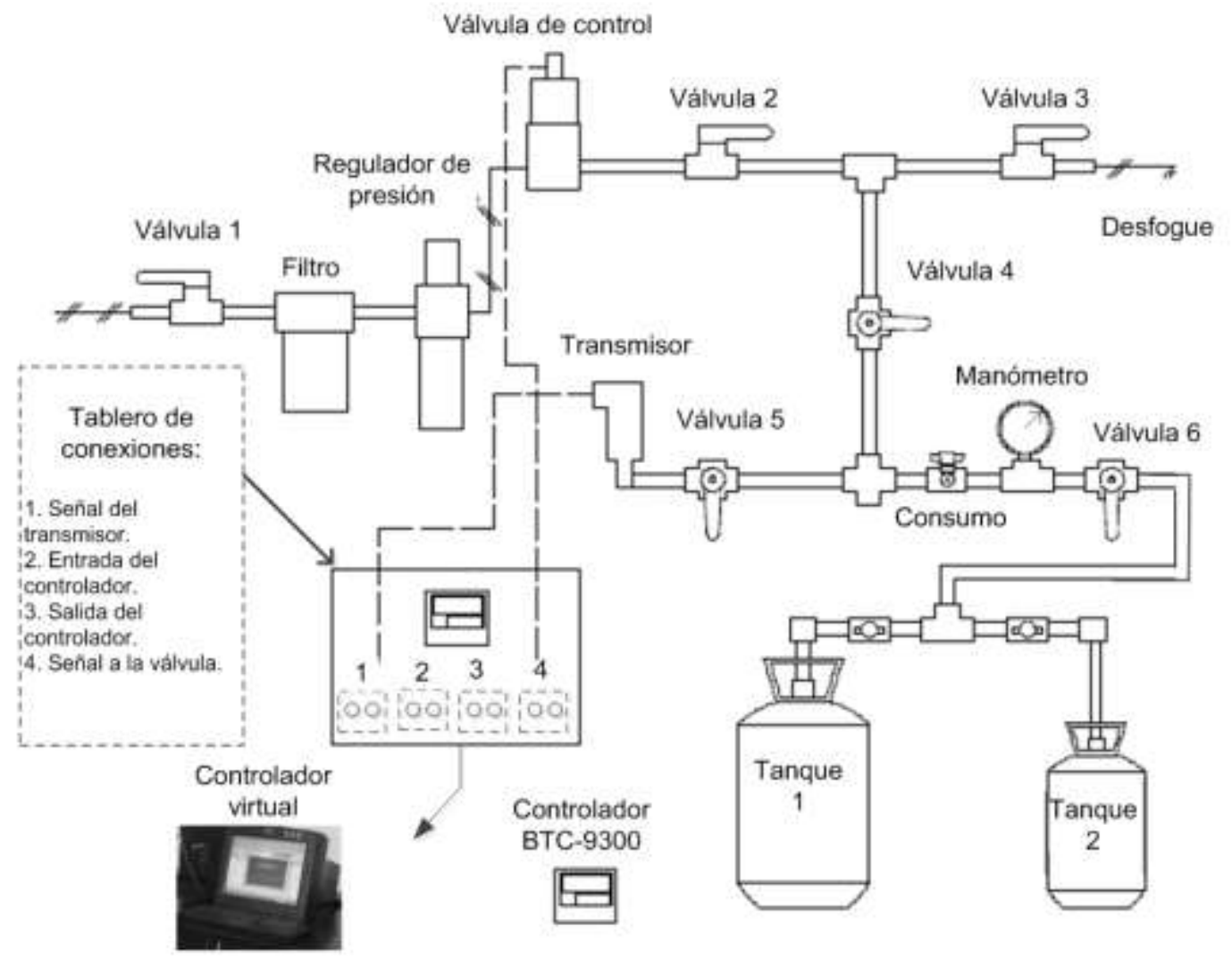

Fig. 2: Diagrama esquemático del sistema de control de presión.

\section{Transmisor de presión}

Para determinar el valor de la presión en los tanques se utilizó el sensor de la marca Green de la serie p250, el cual se presenta en la Figura 3. El uso de materiales cerámicos en el elemento de detección y su montaje proporcionan una excelente resistencia a la mayoría de líquidos y productos químicos. La robustez y fiabilidad son también reforzadas por una carcasa de acero inoxidable para resistir la corrosión. El elemento de sensado contiene un circuito integrado confiable de estado sólido que acondiciona la señal. La salida del sensor proporciona una corriente de $4 \sim 20 \mathrm{~mA}$, que corresponde a una variación de $0 \sim 6 \mathrm{~kg} / \mathrm{cm}^{2}$. 


\section{Válvula de control}

Para regular la presión en los tanques se instaló una válvula de control denominada MPPE-3-1/4-6-420-B de la marca Festo, la cual se muestra en la Figura 4. Es denominada comercialmente regulador de presión proporcional, sus primeras siglas MPPE corresponden al tipo de regulador, un regulador de presión proporcional con válvula de switcheo, -3 son las vias de conexión con el sistema neumático, 1/4 es la medida de conexión para la tuberia, - 6 es el rango de presión en bars que puede regular, -420 es el set point de entrada analógica que corresponde a un rango de 4-20mA. La -B corresponde a la generación o serie de la válvula. Tiene un voltaje de alimentación de $18-30$ volts y un rango de flujo de 350-8800 L/min.

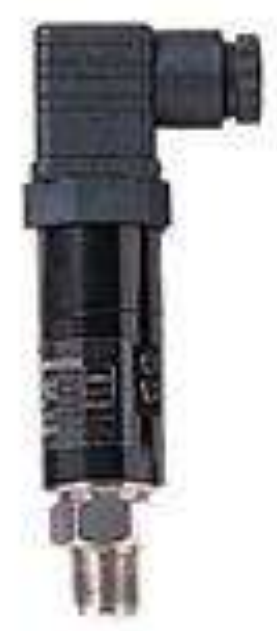

Fig. 3: Sensor Green de la serie p250

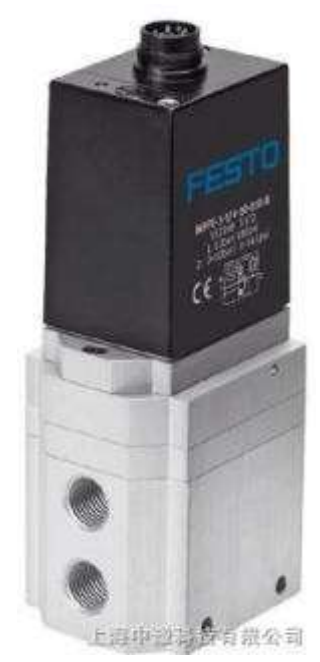

Fig. 4: Válvula MPPE-3-1/4-6-420-B

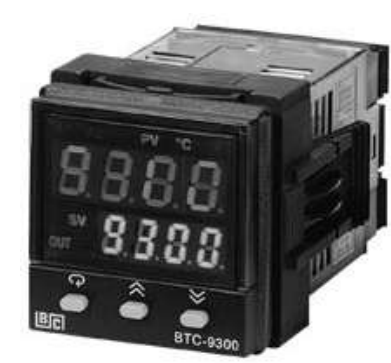

Fig. 5: Controlador de procesos

Básicamente el funcionamiento está basado en el switcheo de 2 válvulas actuadas eléctricamente a través de la señal de entrada, es decir de acuerdo a la corriente de entrada se acciona una u otra válvula de manera proporcional, una de ellas va a un desfogue y la otra a la línea de salida, por lo tanto si se requiere una determinada presión superior a la que existe en el sistema en un momento determinado, la válvula que va a la línea se abre mientras que el desfogue tiende a cerrarse hasta llegar al valor deseado. En el caso contrario cuando se requiere una presión menor el desfogue se abre y la salida tiende a cerrarse.

\section{Controlador digital configurable}

En este proyecto se instaló de manera fija en la estación de trabajo didáctica, el controlador digital configurable BTC-9300, para experimentar con un controlador comercial utilizado en la industria. En la Figura 5 se muestra el controlador. El controlador está basado en un microprocesador que conjunta el control PID + lógica difusa. Con dos pantallas LED, donde se indican los valores del proceso y del punto de ajuste o set point. La tecnología de lógica difusa permite que un proceso pueda alcanzar un punto de ajuste predeterminado en el menor tiempo posible, con un mínimo de picos durante el inicio del proceso.

El controlador se configuró de acuerdo a las condiciones de la señal de control, la variable del proceso y al tipo de señales de instrumentación utilizadas $(4 \sim 20 \mathrm{~mA})$. Para sintonizar el controlador se utilizó la función de autosintonía, ésta automáticamente mide, calcula y ajusta el PID óptimo. El controlador después de este proceso presentó los siguientes valores en los parámetros: banda proporcional $(P)=10$, tiempo integral $(I)=$ 1 y tiempo derivativo $(D)=0.2$.

\section{Controlador virtual}

Con la finalidad de que el sistema de control diseñado sea flexible y adaptable al avance de la tecnología, se hace uso de la computadora para la elaboración de un controlador virtual bajo el lenguaje de programación visual LabVIEW. La Figura 6 muestra el panel frontal del controlador PID, donde se establecen puntos de ajuste, se experimenta con los parámetros del controlador PID, se seleccionan el canal de salida, el canal de entrada y los límites de la tarjeta de adquisición de datos, así como también se pueden observar las gráficas de la variable del proceso, punto de ajuste y salida del PID. 


\section{CONTROLADOR PID PARA LA ESTACIÓN DE PRESIÓN DIDÁCTICA}
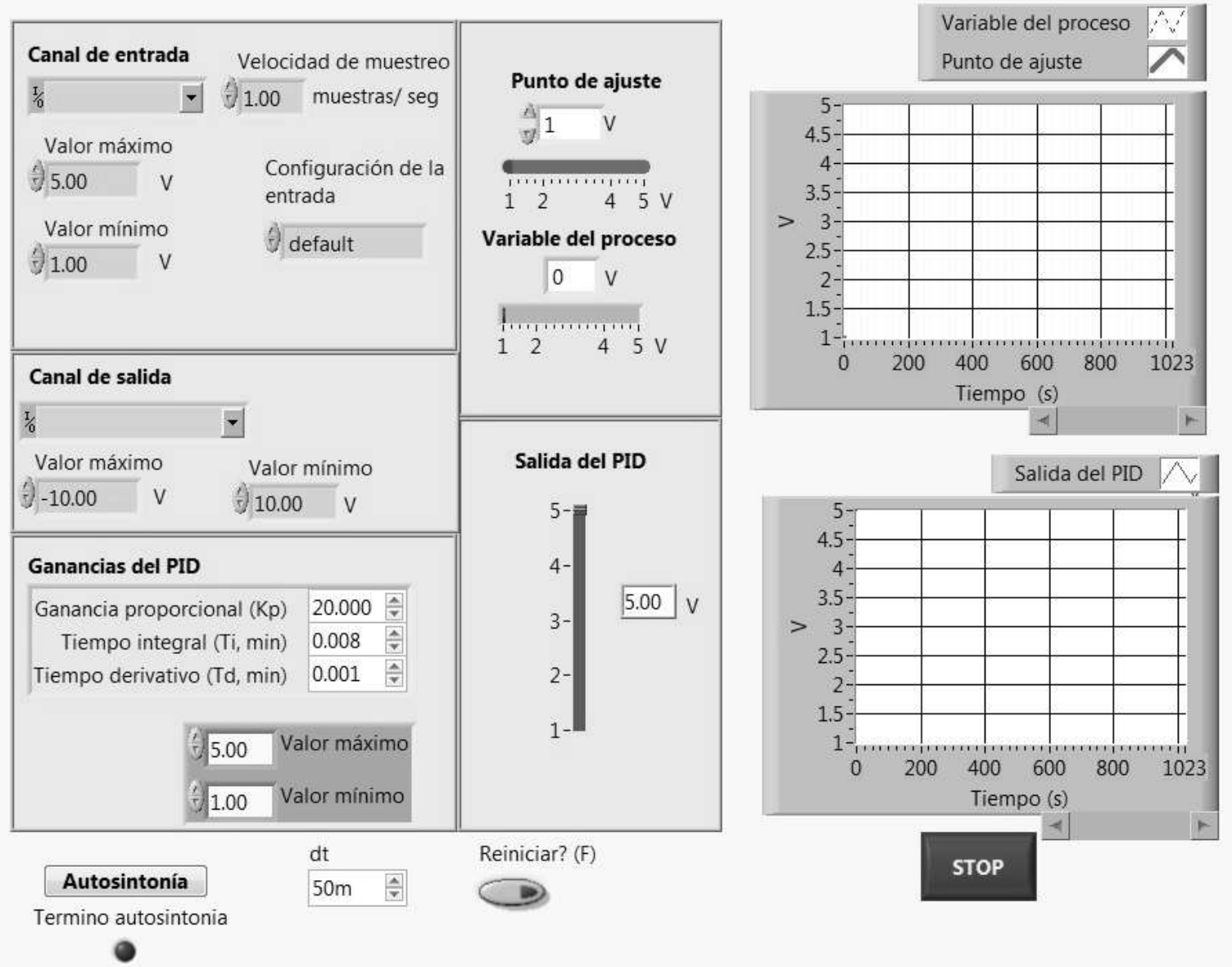

Fig. 6: Interfaz de usuario del controlador virtual.

El controlador virtual implementado, posee la función de autosintonización, la cual se basa en el método de Ziegler y Nichols (Smith y Corripio, 2004). Utilizando esta función se obtuvieron como ganancias $P=2.22$, $\mathrm{I}=0.065$ y $\mathrm{D}=0$.

\section{RESULTADOS Y DISCUSIÓN}

En la Figura 7 se muestra una foto del sistema de control de presión didáctico, donde se observa la conclusión de la implementación física. Se consideraron aspectos de portabilidad para su uso (J. McKay et al., 2009). La parte inferior del sistema didáctico posee 4 ruedas las cuales le proporcionan movimiento, de manera que pueda ser transportada entre los diferentes laboratorios y salones de clases para su uso como sistema de control de presión, o debido a su diseño en arquitectura abierta la utilización de alguno de sus componentes en otra aplicación, cuidando únicamente respetar las especificaciones de las señales de instrumentación. Y así equilibrar o disminuir la cantidad de usuarios en los laboratorios debido al crecimiento de la población estudiantil en la Universidad.

Para demostrar su funcionamiento se pone en operación utilizando el controlador virtual y el controlador digital configurable instalados. Para la conexión entre la computadora y la estación de trabajo se utiliza la tarjeta de adquisición de datos NI PCl-6221 de National Instruments. Los resultados experimentales se presentan a continuación.

\section{Respuesta transitoria del controlador digital configurable}

El controlador BTC-9300 con los parámetros determinados en el proceso de autosintonía, presenta la respuesta que se muestra en la Figura 8 . Para un set point variable en un rango de $1.5 \mathrm{~V}$ a $2.5 \mathrm{~V}$ que corresponde a $1.5 \mathrm{~kg} / \mathrm{cm}^{2}$ y $3.7 \mathrm{k} / \mathrm{cm}^{2}$, respectivamente. 


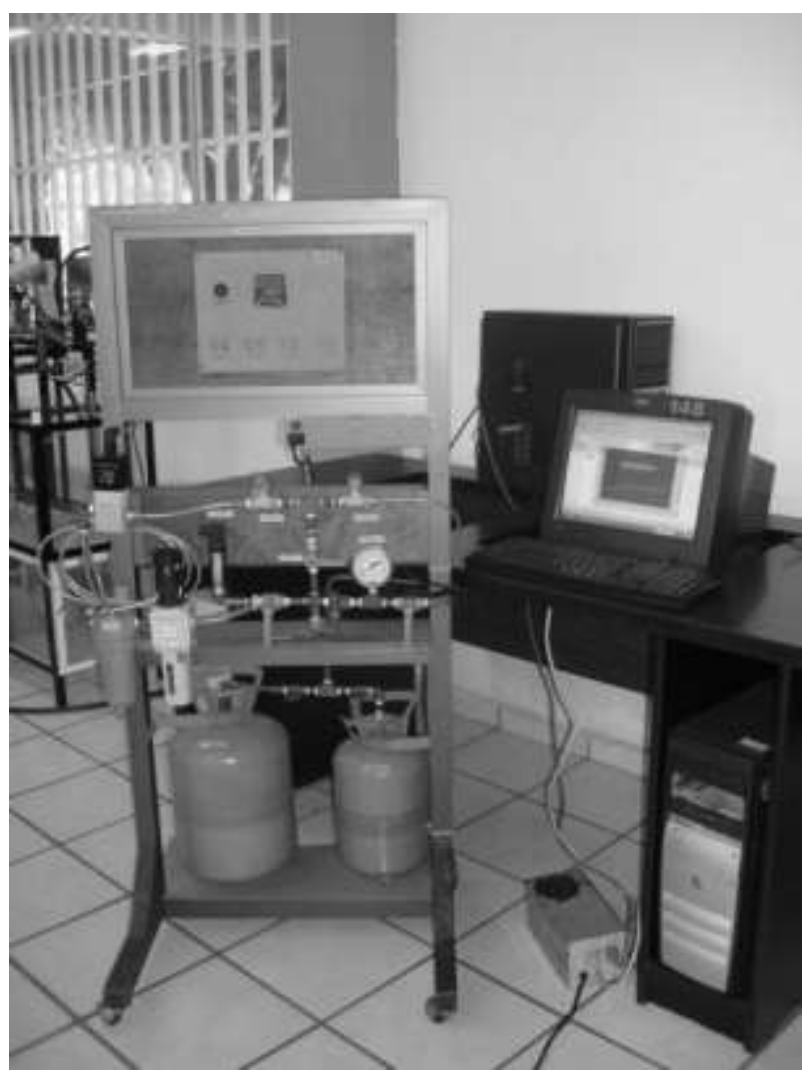

Fig. 7: Presentación física de la estación de trabajo didáctica para la variable presión

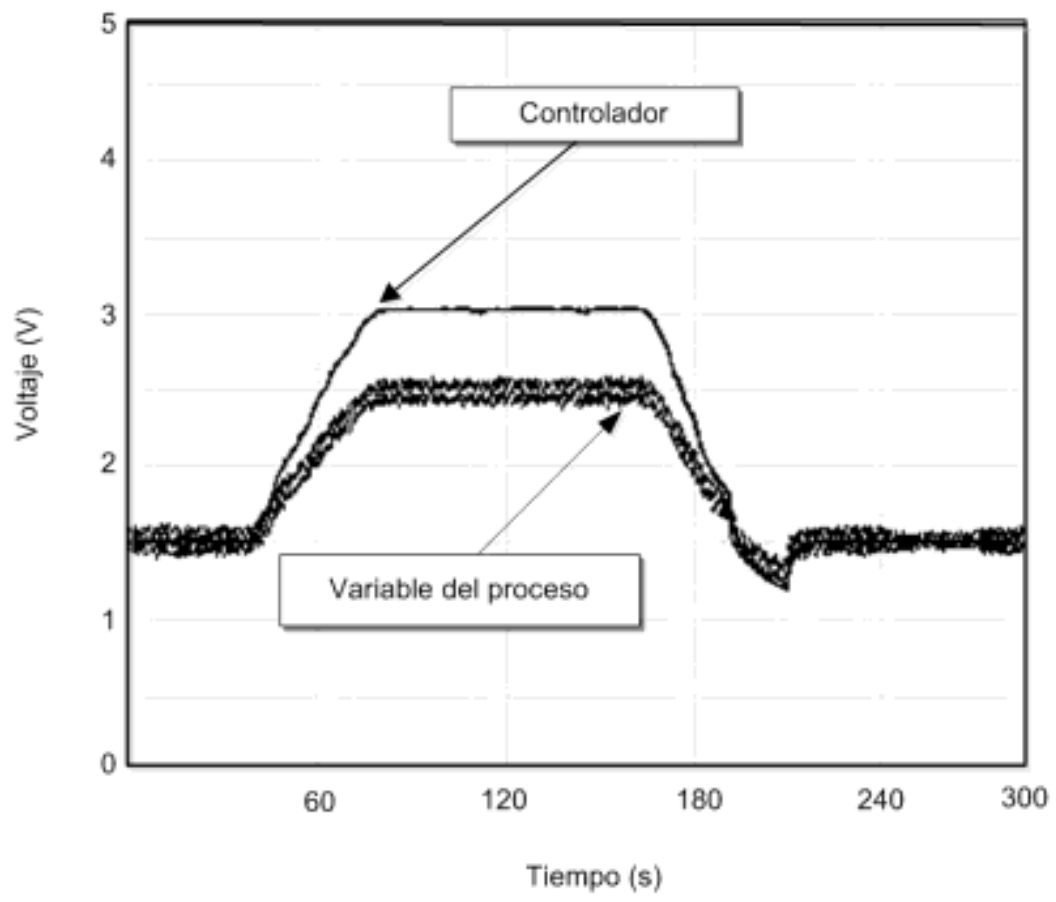

Fig. 8: Controlador digital configurable BTC-9300 con $P=10, I=1$ y $D=0.2$.

Respuesta transitoria del controlador virtual

La Figura 9 muestra las respuestas obtenidas después del proceso de autosintonía del controlador virtual. Para un set point de $1.5 \mathrm{~V}\left(1.5 \mathrm{~kg} / \mathrm{cm}^{2}\right)$ a $2.5 \mathrm{~V}\left(3.7 \mathrm{~kg} / \mathrm{cm}^{2}\right)$ y viceversa. 

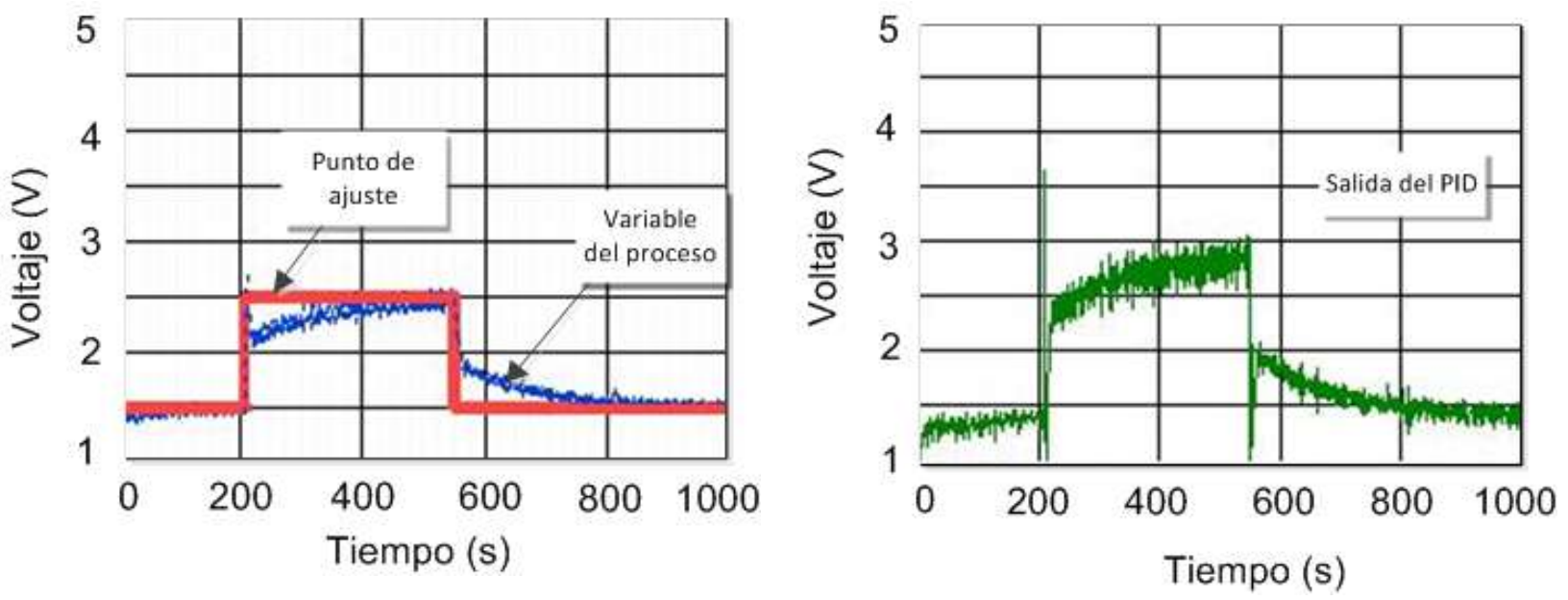

Fig. 9: Respuestas del controlador virtual con $P=2.22, I=0.065$ y $D=0$.

La dinámica de las respuestas transitorias obtenidas del sistema de control de presión didáctico es bastante comparable a los simuladores y modelos de laboratorio comerciales (Festo-didactic; Tecquipment), Por lo que demuestra ser un equipo de laboratorio que permite fortalecer la formación de competencias en estudiantes del área de instrumentación y control. De forma que las diferentes actividades realizadas con él, permitirán predecir el resultado de las acciones que se tomen sobre el proceso o sistema de control, comprender por qué los eventos ocurren, explorar los efectos de las modificaciones, evaluar ideas y su viabilidad, así como estimular el pensamiento creativo.

\section{CONCLUSIONES}

La estación de trabajo didáctica está diseñada e implementada en arquitectura abierta, logrando un acoplamiento entre software y hardware, obteniendo un sistema flexibilidad abierto a futuros desarrollos, lo que permite la enseñanza y la investigación en temáticas tales como: registro de señales, ajuste de controladores, diseño de controladores, prueba de controladores (analógicos, digitales, inteligentes, etc.), instrumentación clásica, instrumentación virtual, caracterización de procesos, configuración de sensores, calibración de actuadores, etc.; ventaja que presenta a diferencia de algunas que se encuentran en el mercado, que tienen el inconveniente de ser sistemas cerrados, difíciles de modificar, con un costo mayor y con tendencia a hacerse obsoletas (Charre et al., 2009).

El presente trabajo genera la creación de más productos de investigación como tesis de licenciatura o maestría, tales como un manual de prácticas de instrumentación y control, el control y supervisión de la estación a través de Internet, entre otras. Actualmente, está siendo utilizada por los estudiantes para la realización de prácticas y tesis enfocadas a la comparación de diversos algoritmos de control. Por otra parte el controlador virtual implementado no únicamente está limitado para aplicarse al control de la variable presión de la estación de trabajo didáctica, también se puede aplicar al control de diferentes procesos que manejen las señales estándar de instrumentación (4 20 mA y 1 5 V).

\section{AGRADECIMIENTOS}

Los autores agradecen el apoyo a la Facultad de Ingeniería Electromecánica de la Universidad de Colima.

\section{REFERENCIAS}

Alam F., R. G. Hadgraft, A. Subic; Technology-Enhanced Laboratory Experiments in Learning and Teaching, Using Technology Tools to Innovate Assessment, Reporting, and Teaching Practices in Engineering Education, 289-302. doi:10.4018/978-1-4666-5011-4.ch021 (2014).

Charre S., J. Gudiño, R. Martínez, S. Campos, Sistema de Control de Nivel de Líquido, Congreso Interamericano de Computación Aplicada a la Industria de Procesos 2009, Montevideo Uruguay, Agosto (2009). 
Festo, Estación de trabajo compacta MPS® PA con tramos de regulación de nivel, caudal, presión y temperatura, http://www.festo-didactic.com/int-es/learning-systems/automatizacion-de-procesos/. Acceso: 1 de agosto (2014).

Fredes, C.A., J. P. Hernández, D. A. Díaz; Potencial y Problemas de la Simulación en Ambientes Virtuales para el Aprendizaje, Formación Universitaria: ISSN: 0718-5006, vol. 5 (1), 45-56 (2012).

Guevara, P., J. S. Falcón, R. J. Sandoval, J. J. Medel; Equipos Didácticos Industriales en el modelo educativo de los Cecati, Innovación Educativa: ISSN: 1665-2673, vol. 9 (48), 73-81 (2009).

Márquez D.A., O. Cárdenas; Implementación de un Laboratorio Virtual para la enseñanza de Controladores PID, Información Tecnológica: ISSN: 0718-0764, vol. 19(3), 75-78 (2008).

Matijević, M., M. Stefanović, V. Cvjetković, V. Joković, N. Babajić, M. Ravlić, S. Nestic; The Development and Implementation of a Thermal Process Trainer for Control and Measurement via the Internet, Computer Applications in Engineering Education: ISSN: 1099-0542, vol. 22 (1), 167-177 (2014).

McKay, J., I. Kovacevich, T. Philpott, R. McKay, B. Rhinehardt, D. Lee, Portable Workstation, US 2009/0200902A1, 13 de Agosto (2009).

Pallás, R., Sensores y Acondicionadores de Señal, Alfaomega Marcombo (2008).

Smith, C. A. y A. B. Corripio, Control Automático de Procesos, 266-285, Limusa, México (2004).

Świder J., P. Michalski, and G. Wszołek; Laboratory support for the didactic process of engineering processes automation at the Faculty of Mechanical Engineering, Journal of Achievements in material and manufacturing engineering, Vol. 15 (2006).

Tecquipment, Medición de flujo y presión, http://www.nuevosrecursos.com/index.php/mecanica-de-fluidos/. Acceso: 1 de agosto (2014). 